\title{
Endobronchial tuberculosis in an immunocompetent young filipino male - A case report from Kingdom of Saudi Arabia
}

\author{
Abdul Majid Arwadi ${ }^{1}$, Farhan Khalid ${ }^{2 *}$, Sara Sadiq ${ }^{3}$, Naveed Qureshi ${ }^{4}$ and Usman Ali Maitla $^{4}$ \\ ${ }^{1}$ MD Pulmology and Critical Care, MD Internal Medicine, Department of Internal Medicine, Specialized Medical Center Hospital, Riyadh, Saudi Arabia \\ ${ }^{2}$ MBBS, MRCP, Adult Hematology, Department of Oncology, King Abdulaziz Medical City, Riyadh (KAMC-RYD), Ministry of National Guard, Saudi Arabia \\ ${ }^{3}$ MBBS, M.Phil. Department of Physiology, Ziauddin University Clifton, Karachi, Pakistan \\ ${ }^{4}$ MBBS, Adult Hematology, Department of Oncology, King Abdulaziz Medical City, Riyadh (KAMC-RYD), Ministry of National Guard, Saudi Arabia
}

\begin{abstract}
Endobronchial tuberculosis (EBTB), is a tuberculous infection of the tracheobronchial tree. It is common among young adults and specifically females. It is a poorly understood phenomenon and diagnosis is frequently delayed as sputum smears are often false negative for acid fast bacilli with no clinical indicators, resulting in delayed diagnosis. It is a challenge to diagnose EBTB, as the chest X-ray in EBTB could appear to be normal and diagnostic confirmation can only be achieved by bronchoscopy along with biopsy and lavage sample. EBTB presenting as middle lobe syndrome (MLS) has rarely been documented in west is also a rare finding in Asia. Its early detection and prompt treatment can prevent complication.

To the best of our knowledge, none of the study had reported the Patients with EBTB in Kingdom of Saudi Arabia. We hereby report a case of EBTB involving right middle and lower lung lobes in an immunocompetent young Filipino male.
\end{abstract}

\section{Introduction}

Tuberculosis (TB) is a global challenge, with high incidence and mortality rate. It has become a major cause of mortality alongside HIV [1]. Worldwide, 9.6 million people are estimated to have fallen ill with TB in 2014. Prompt diagnosis and treatment of TB saved about 43 million lives between year 2000 and 2014 [2]. An estimated of about $64,345 \mathrm{~TB}$ cases in Kingdom of Saudi Arabia over the period of 20 years, among them $48 \%$ were non-Saudis [3].

Tuberculous infection of the tracheobronchial tree is known as endobronchial tuberculosis (EBTB). It is a challenge to diagnose EBTB, as the chest X-ray in EBTB could appear to be normal and diagnostic confirmation can only be achieved by bronchoscopy along with biopsy and lavage sample. This variant of tuberculosis prevalent and highly infectious [4-7]. The Middle lobe syndrome (MLS) secondary to tuberculosis is a distinct rarity with very few cases reported [4]. The term MLS is first described by Graham et.al, he reported a series of 12 cases of middle lobe atelectasis which were non-tuberculous origin [8]. MLS is more common than it is perceived. Surender et.al in his study mentioned the difficulties that are encountered in diagnosing MLS, because of the presence of non-specific symptoms even after accurate diagnosis is established, the clinical course is variable with complications. It is usually seen in second and third decade and has more predilections towards females [9].

To the best of our knowledge, none of the study had reported the Patients with EBTB in Kingdom of Saudi Arabia. We hereby report a case of EBTB involving right middle and lower lung lobes in an immunocompetent young Filipino male.

\section{Case report}

\section{Presentation}

A 31-year-old immunocompetent Filipino male, healthcare professional by occupation, presented in Emergency department with complaint of cough with sputum, fever and shortness of breath for 1 week.

The cough was productive with white color sputum but not associated with hemoptysis. Fever was of intermittent type, low in grade but was not associated with chills and rigors and relieved by taking antipyretics. The patient had progressive shortness of breath which was aggravated by doing physical activity and relieved by taking rest. His symptoms were of short duration of 1 week and there was no history of weight loss, anorexia or night sweats. He had no family history of tuberculosis or no history of contact with any patient who was known to have tuberculosis, but his profession made him a high risk.

\section{Examination}

On clinical examination he was thin in built, well-oriented and lying comfortably on bed. General physical examination revealed no significant sign of pallor, cyanosis, jaundice, clubbing or peripheral

${ }^{\star}$ Correspondence to: Farhan Khalid, MBBS, MRCP, Adult Hematology, Department of Oncology, King Abdulaziz Medical City, Riyadh (KAMC-RYD) Ministry of National Guard, Saudi Arabia, E-mail: farhan253@gmail.com

Key words: Tuberculosis, Endobronchial Tuberculosis, Middle Lobe syndrome

Received: September 19, 2018; Accepted: September 27, 2018; Published: September 29, 2018 
lymphadenopathy. His respiratory rate was 20 breaths/min. On respiratory system examination, trachea was centrally located. He had asymmetrical chest expansion and decreased vocal fremitus at middle and lower lobes of the right lung. There was dull percussion note at the same site. On auscultation, vesicular breath sound was diminished with decreased vocal resonance specially at the middle and lower lobes of the right lung. The remaining systematic examinations were normal.

\section{Investigations}

His laboratory investigations revealed mildly elevated WBC and Neutrophils count with normal lymphocytes. His Hepatic and renal function tests were within normal range. Chest $\mathrm{x}$-ray showed middle and lower lobes of the right lung filled with airspace shadowing with pleural effusion as shown in (Figure 1).

Corona swab for Influenza-A virus (H1N1) was negative. Later CT Chest was performed which showed consolidation of the middle and lower lobes of the right lung in addition to air bronchogram and small amount of pleural effusion. The sputum sample was negative for Acid Fast bacilli. A repeat CT scan was arranged, and it showed the same findings with an increase in pleural effusion as presented in (Figure 2).

Eventually bronchoscopic biopsy and broncho-alveolar lavage was arranged. The biopsy revealed a startling finding of marked/diffuse granulomatous infiltrations with several well-formed granulomas along with epithelioid histiocytes and Langhans-type giant cells. Necrosis was seen in the center of each granuloma. AFB staining was again negative for acid fast bacilli. However, the histopathology was consistent with tuberculosis. On the other hand, bronchoalveolar Lavage sample sent for Acid Fast Bacilli DNA amplification came positive for $M$. Tuberculosis complex DNA. Acid Fast Bacilli Culture also confirmed the presence of tuberculosis.

\section{Treatment}

Initially he was admitted in the hospital as a case of pneumonia. As Corona swab for $\mathrm{H} 1 \mathrm{~N} 1$ was negative therefore, empirically he was

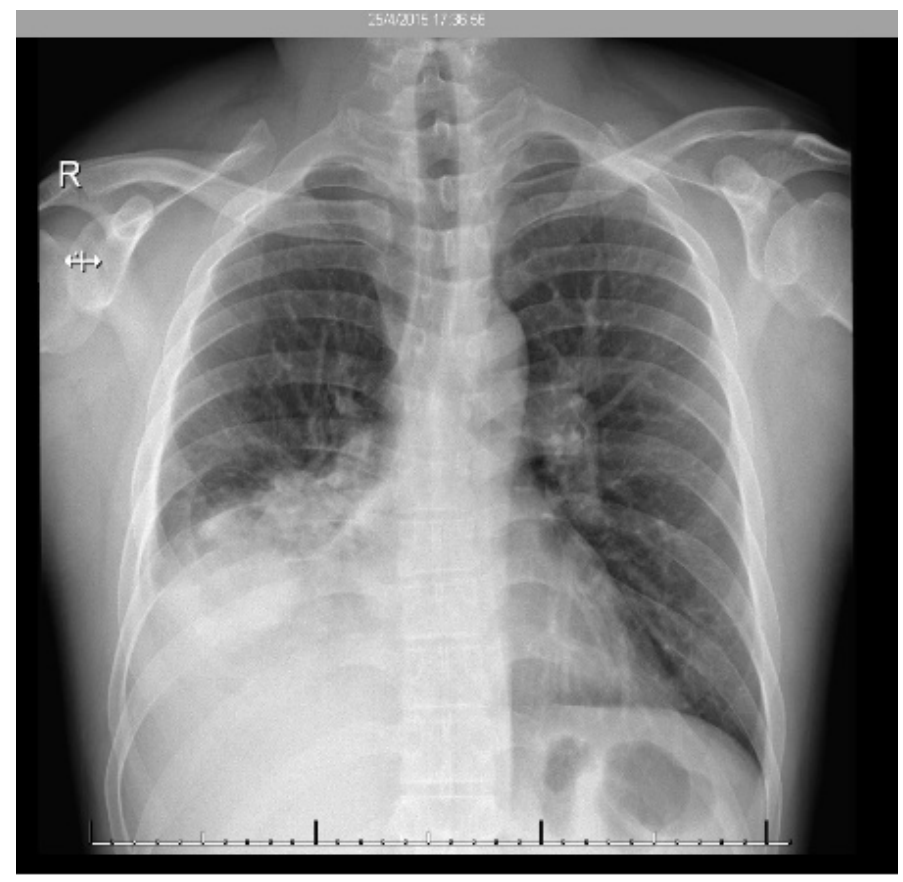

Figure 1. Pleural effusion at middle and lower lobes of the right lung

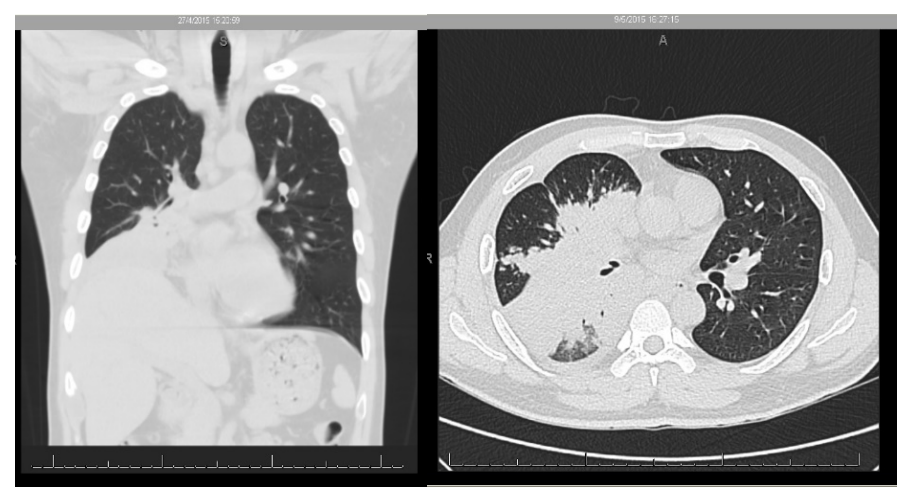

Figure 2. CT scan of thorax showing Pleural effusion

started on levofloxacin to which; he did not show much favorable response. Subsequently Imipenem was added to his regimen.

Clinically the patient showed no improvements, vancomycin was also added to his treatment regimen. Even after that the patient did not showed much improvement in his symptoms and continued to have fever and progressive shortness of breath. After reviewing the investigation reports, the anti-tuberculosis medications were prescribed, which were Isoniazid, Rifampicin, Ethambutol and Pyrizinamide.

Within the next few days, the patient reported a gradual betterment in his condition with resolution of fever within 48 hours after starting anti-tuberculosis treatment. He was eventually discharged on antituberculosis medications and he reported significant improvement in his overall condition.

\section{Discussion}

Endobronchial tuberculosis (EBTB) continues to remain a challenge [6,9]. It has variable features as the characteristic symptoms of EBTB including anorexia, weight loss and night sweats are not seen in any patient. Middle lobe syndrome occurs in all age groups and is divided into an obstructive type, with a demonstrable airway occlusion, and a non-obstructive type, with a patent right middle lobe bronchus apparent on bronchoscopy $[4,9,10]$. The Pathogenesis of EBTB has not been fully understood [4,9]. However, literature reveals many possible mechanisms such as direct extension from an adjacent parenchymal focus, haematogenous dissemination, lymph node and spread of infection via the lymphatic [9].

Persistent cough although is a common finding, was not present in our case. Roy et al reported 3 cases of unresolved pneumonia with sputum smear negative for acid fast bacilli were diagnosed through bronchial biopsy [10]. This finding is similar to our case report. Literature emphasized on the variability of symptoms. It is manifested that the symptoms which are classical for $\mathrm{TB}$, may be present but will not be characteristic [9]. Fever, cough, and dyspnea were the prominent complains of our patient while hemoptysis, weight loss and anorexia were not present which led to diagnostic difficulty. Sputum examination although the first step has a low diagnostic yield $[9,11]$. In one of the studies, it was noticed that 23 diagnosed case of EBTB from biopsy had negative sputum sample [12]. The role of tuberculin test although emphasized; has less specificity, while CT scan has improved sensitivity. CT chest can be useful in detecting bronchial stenosis which is a complication of EBTB [13]. On the other hand, bronchoscopy and biopsy is mandatory for the diagnosis of EBTB. Histopathological findings aid in the early diagnosis and prompt starting of treatment 
[12]. This was certainly true with our case as well where the diagnosis was confirmed by biopsy through histopathological finding and treatment was started which was later re-affirmed by the Broncho alveolar Lavage sample. Pulmonary function test (PFT) also has a role in the diagnostic process, individuals with EBTB present with a predominantly restrictive pattern [6]. But PFT was not performed for our patient as his symptoms were nonspecific for TB.

The treatment of EBTB is the same as pulmonary tuberculosis [9]. It involves four primary drugs which include, Isoniazid, Rifampicin, Ethambutol and Pyrazinamide along with Streptomycin. In drug resistant cases, treatment must be based on susceptibility results. The clinical course depends on the histopathological finding; out of these subtypes; the actively caseating type is reported as the most common form while the ulcerative type as the least common one. These Histopathological classifications of EBTB are closely related to the extent of disease progression [12]. The role of surgery has been considered in treatment refractory cases. Interventional bronchoscopy is another alternative treatment strategy to surgical resection in the management of stenosis [9].

\section{Conclusion}

In conclusion, EBTB is a rare manifestation of TB, its mechanism is not fully understood. Bronchoscopy and histopathology are the mainstay of diagnosis, sputum smear is mostly negative, CT scan can be helpful in reaching the diagnosis. There are no case reports of such cases in Saudi Arabia. We believe that early detection and prompt treatment can prevent complication.

\section{References}

1. Gupta PP, Gupta K, Agarwal D (2006) Middle lobe syndrome due to tuberculous etiology: a series of 12 cases. Indian journal of tuberculosis 53: 104.
2. Organization WH (2015) Global tuberculosis report. World Health Organization

3. Al-Orainey I, Alhedaithy MA, Alanazi AR, Barry MA, Almajid FM (2013) Tuberculosis incidence trends in Saudi Arabia over 20 years: 1991-2010. Annals of thoracic medicine 8: 148. [Crossref]

4. Garg T, Gera K, Shah A (2015) Middle lobe syndrome: an extraordinary presentation of endobronchial tuberculosis. Advances in Respiratory Medicine 83: 387-391. [Crossref]

5. Chung HS, Han SK, Shim YS, Kim KY, Han YC, et al. (1991) Balloon dilatation of bronchial stenosis in endobronchial tuberculosis. Tuberculosis and Respiratory Diseases 38: 236-244.

6. Lee J, Chung H (2000) Bronchoscopic, radiologic and pulmonary function evaluation of endobronchial tuberculosis. Respirology 5: 411-417. [Crossref]

7. Um S, Yoon Y, Lee S, Yim J, Yoo C, et al. (2008) Predictors of persistent airway stenosis in patients with endobronchial tuberculosis. The international journal of tuberculosis and lung disease 12: 57-62. [Crossref]

8. Graham EA, Burford TH, Mayer JH (1948) Middle lobe syndrome. Postgraduate medicine 4: 29-34.

9. Kashyap S, Solanki A (2014) Challenges in endobronchial tuberculosis: from diagnosis to management. Pulmonary medicine 594806.

10. Roy PP, Dey SK, Sarkar A, Dwari AK, Banerjee A, Banerjee R (2010) Diagnosis of three cases of endobronchial tuberculosis presenting as unresolved pneumonia, following fiberoptic bronchoscopic biopsy. Lung India: official organ of Indian Chest Society 27: 185-188. [Crossref]

11. Lee KS, Yoon JH, Kim TK, Kim JS, Chung MP, et al. (1997) Evaluation of tracheobronchial disease with helical CT with multiplanar and three-dimensional reconstruction: correlation with bronchoscopy. Radiographics 17: 555-567.

12. Ozkaya S, Bilgin S, Findik S, Kok HC, Yuksel C, et al. (2012) Endobronchial tuberculosis: histopathological subsets and microbiological results. Multidisciplinary respiratory medicine $7: 34$.

13. Yu W, Rong Z (1999) Clinical analysis of 90 cases with endobronchial tuberculosis. Zhonghua jie he he hu xi za zhi= Zhonghua jiehe he huxi zazhi. Chinese journal of tuberculosis and respiratory diseases 22: 396-398.

Copyright: (C2018 Arwadi MA. This is an open-access article distributed under the terms of the Creative Commons Attribution License, which permits unrestricted use, distribution, and reproduction in any medium, provided the original author and source are credited. 